\title{
REVIEW OVER HOLE HEALING TECHNIQUES IN WIRELESS SENSOR NETWORKS
}

\author{
Latesh Mehta and Manik Gupta \\ Department of Computer Science, \\ Chitkara University, Himachal Pradesh, India
}

\begin{abstract}
Improving coverage and connectivity is a very important issue in wireless sensor networks. The unattended or uncovered region in a sensing field is called a coverage hole. A coverage hole impacts the performance of the wireless sensor network, disconnects the network topology and causes delay in data transmission. These coverage holes can be healed randomly or each hole can be assigned a priority value for healing. In this paper we will discuss about various hole healing strategies and their mechanisms.
\end{abstract}

\section{KEYWORDS}

Connectivity, Coverage, Coverage Holes, Hole Healing, Wireless Sensor Network.

\section{INTRODUCTION}

A wireless sensor consists of a sensing component, on-board processing, communication, and storage unit. Many sensors cooperatively monitoring a large physical environment, form a wireless sensor network (WSN)[7]. The wireless sensor networks have different usage in various fields and applications like monitor and protect civil infrastructure, underwater monitoring[16], monitor large geographic areas, volcano monitoring, collecting structural health information etc. In [10] these applications are classified into monitoring and tracking applications e.g. health and wellness monitoring, power monitoring, inventory location monitoring, tracking objects, animals, humans, and vehicles. Sometimes, sensors are deployed in very remote and inaccessible areas; therefore the task of maintaining the coverage and connectivity becomes a major issue in WSN. The uncovered region in the target sensing field is considered as a coverage hole. Coverage holes can be introduced as a result of a node failure or initial deployment. Fig.1(a) shows a 2dimentional image of a field which shows coverage holes introduced as a result of initial deployment. Fig. 1(b) shows coverage holes in a field introduced as a result of node failure.

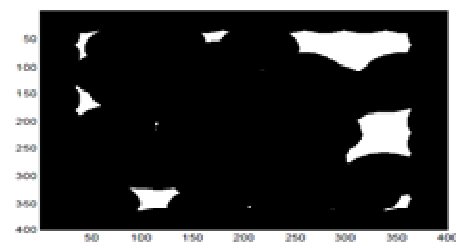

(a)

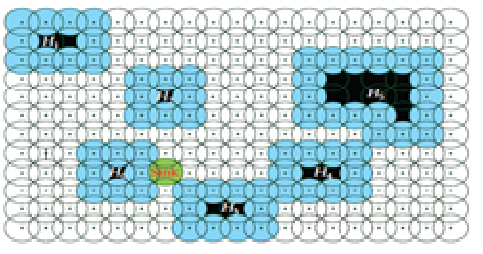

(b)

Figure 1. An example of coverage holes in a target sensing field. (a) 2-dimentional image showing coverage holes as a result of initial deployment. The white area in the graph shows the uncovered region in the field.[4] (b) Coverage holes as a result of node failure. Hi represents coverage holes in the field.[6]

Sensor nodes must be self-managing[7] and should discover, identify, and react to network disruptions. To improve the coverage and maintain the network connectivity various strategies are DOI : 10.5121/ijasuc.2016.7101 
proposed in recent years. In energy-constrained sensor networks, these solutions must be implemented in such a way that they do not incur excessive energy overheads. In [9] coverage problem is classified into three categories i.e. area coverage, point coverage and barrier coverage. In PEAS mechanism[11], based on node density detection, duty cycling is used where some nodes are allowed to sleep and nodes are awakened at random time interval. In [12] unnecessary sensing work is avoided by detecting redundant nodes and sending them to a sleep state. The work in [13] compares energy efficiency of the sensor network with fixed sensing range and the network consisting of nodes with adjustable sensing range. G-MSC [14], a target coverage algorithm, constructs continuous node sets to cover as many targets in the network. The coverage algorithm in [15] relies only on 3 hop neighbors for its information. In[17], holes are classified into 5 types, namely, coverage hole, routing hole, jamming hole, sink/black hole and worm hole. The study in[17] also discuss detection of hole using voronoi and triangular diagram approach. All these studies focuses mainly on the hole detection, but we must not forget after hole detection the next phase i.e. hole healing is also very important. Healing a coverage hole not only improves coverage but it also improves the connectivity of the network. The complete hole healing process can be divided into two parts:Hole Selection and Healing Process.

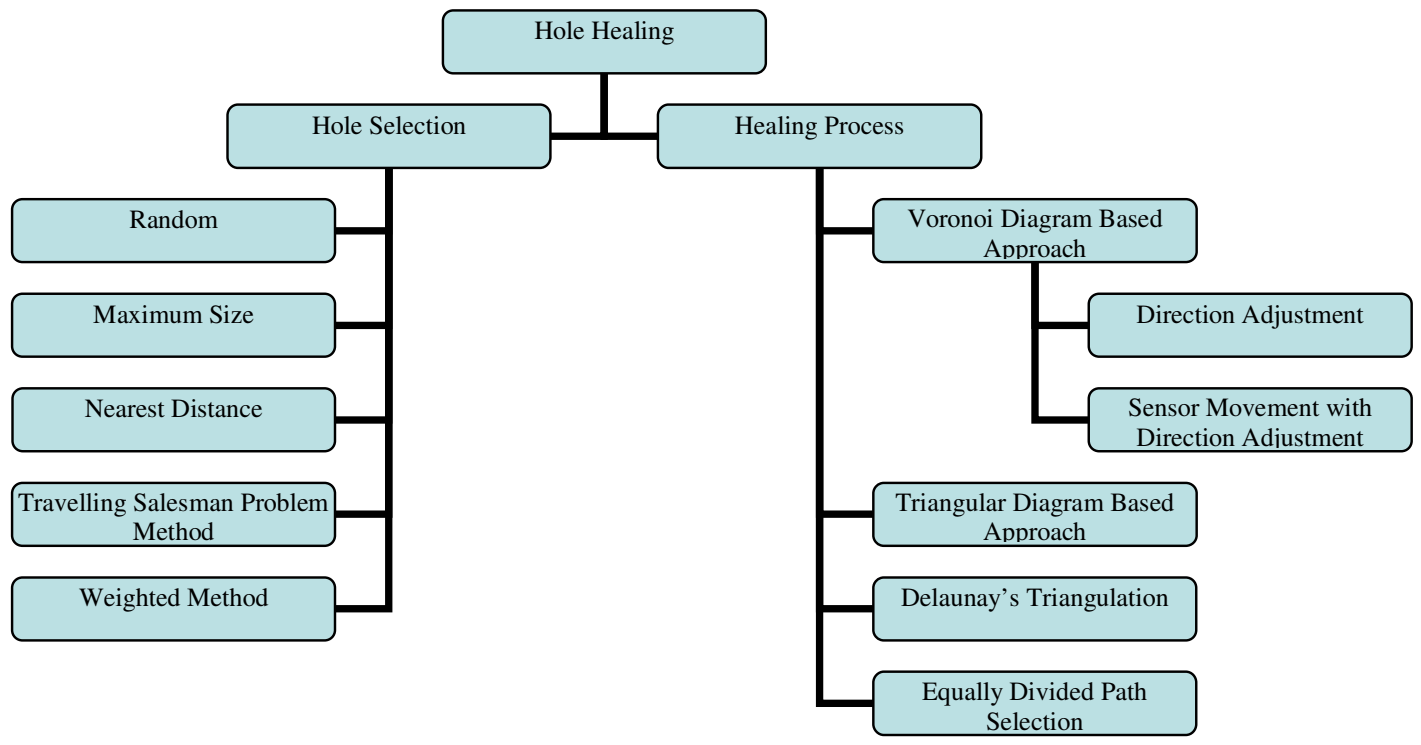

Figure 2. Classification of Hole Selection and Healing Process.

The hole selection process decides which hole should be selected first for healing and the healing process tells how the selected hole should be healed to ensure maximum coverage and connectivity. Fig. 2 shows various types of hole selection methods and healing process. In the rest of the paper section 2 will give details about various hole selection methods, section 3 will discuss about healing techniques and section 4 will conclude the paper.

\section{Hole SELECTION METHODS}

The hole selection process is very important during the network maintenance process. Coverage holes in a network are of different shape and size. The effect of their presence in the network is also different and it depends on various factors such as shape and size of the hole, shape and size of the target sensing field, location of the hole and the sink etc. Based on these factors some holes affect the coverage, some affects the connectivity and most of them affect both. The hole which is disturbing network the most, should be selected for healing at the first place. Based on their effect on coverage and connectivity, holes should be assigned a priority value for healing. In recent years, researchers have adopted different methods for hole selection. These methods are as follows: 


\subsection{Random}

The coverage holes are selected on a random basis. This method increases the coverage but it does not optimize the results. It is not a good method for hole selection.

\subsection{Maximum Size}

In this method coverage holes are selected based on the size of the hole. The hole larger in size will have maximum effect on the coverage of the network. Therefore hole with maximum size is selected for healing process. This method improves coverage but has better impact on connectivity than random method.

\subsection{Nearest Distance}

In a wireless sensor network, nodes communicate with the sink through a multihop network. Each node passes message coming from other node towards the sink. Fig. 3 shows data flow towards the sink in a wireless sensor network. Here, nodes closer to the sink will have more data to relay towards the sink. Hence they are more likely to lose their energy as compared to other nodes in the network and if the hole occurs near the sink due to node energy depletion it will induce a delay in data transmission because the messages will have to re-route now to reach the sink. Therefore, the nearest distance method selects holes closer to the sink for healing, to balance the traffic load and to reduce the delay in data transmission.

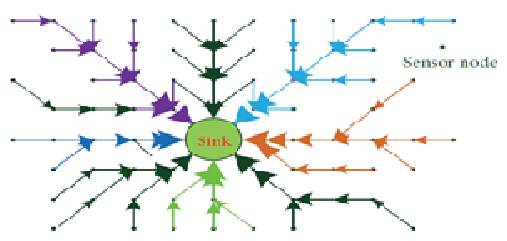

Figure 3. The multi-to-one data flow concept.[6]

\subsection{Travelling Salesman Problem}

There can be different number of holes at different position of time in a wireless sensor network. The hole healing strategy always aim to cover all the holes present in the network for a given interval of time. Therefore, in some hole healing techniques the hole selection procedure is transformed into travelling salesman problem[4]. If the network is using an external source to deploy nodes such as mobile robot, unmanned aerial vehicle(UAV) or other kind of resource, then its path is computed using travelling salesman problem in which a shortest path is computed where every hole is covered only once starting from an origin and returns to its initial position at last. Fig. 4(a) shows the center coordinates of coverage holes in a target sensing field and fig. 4(b) shows the optimal path obtained. This method ensures that every hole is covered once within less time.

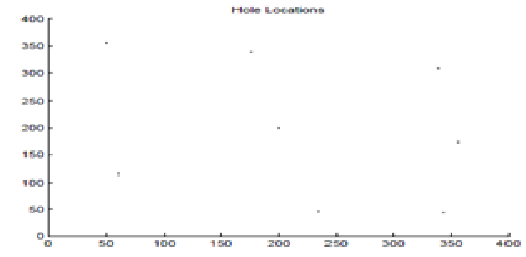

(a) Center Coordinates of hole[4]

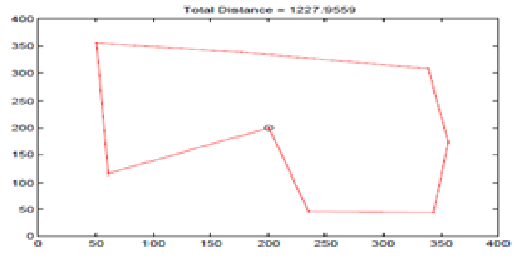

(b) Optimal Path Trajectory[4]

Figure 4. Hole Selection Procedure converted into Travelling Salesman Problem.[4]

\subsection{Weighted Method}

This is the most effective hole selection methods available so far. This method not only improves coverage but it also improves the connectivity. Holes present in the network are given some 
priority for healing and their priority is based on three properties of the coverage hole. These three properties are as follows[6]:

- Hole Angle Metric: Hole angle tells about the width of the hole. In a wireless sensor network data is relayed from all sides towards the sink as shown earlier in fig. 3. A wider hole will hinder data flow towards the sink. The effect on the data transmission is directly proportional to the width of the hole. Therefore, a hole with greater hole angle will have more effect on data transmission. Fig. 5 shows how to calculate hole angle. In fig. $5 \mathrm{H}_{\mathrm{i}}$ represents hole, $\mathrm{B}_{\mathrm{j}}$ represents the hole border node, $L_{j}$ is a ray starting at sink and extends towards $B_{j}$. The ray $L_{j}$ is obtained for every hole border node $B_{j}$ and its slope is calculated. The hole border node $B_{j}$ with maximum slope is represented by $B_{u}$ and $B_{j}$ with minimum slope in represented by $B_{d}$. The angle between these two rays $\left[\mathrm{SINK}, \mathrm{B}_{\mathrm{u}}\right)$ and $\left[\mathrm{Sink}, \mathrm{B}_{\mathrm{d}}\right)$, represented by $\Theta\left(\mathrm{H}_{\mathrm{i}}\right)$ is called the hole angle and it is calculated as[6]:

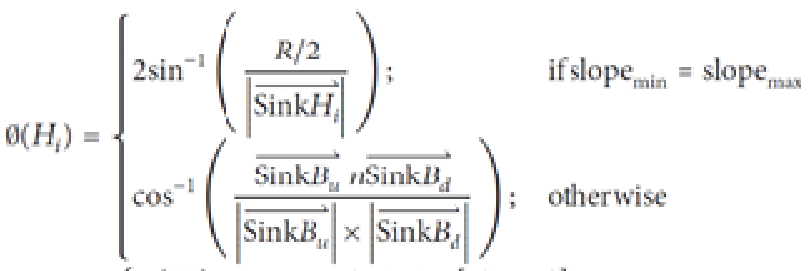

$$
\begin{aligned}
& \Theta\left(H_{i}\right)= \begin{cases}0\left(H_{i}\right) ; & \text { if } \emptyset\left(H_{i}\right) \in\left[0^{\circ}, 180^{\circ}\right] \\
360^{\circ}-0\left(H_{i}\right) ; & \text { if } \emptyset\left(H_{i}\right) \in\left[180^{\circ}, 360^{\circ}\right]\end{cases}
\end{aligned}
$$

Here, $\left[\mathrm{SINK}, \mathrm{H}_{\mathrm{i}}\right.$ ) represents ray starting at sink and extends towards the center of hole.

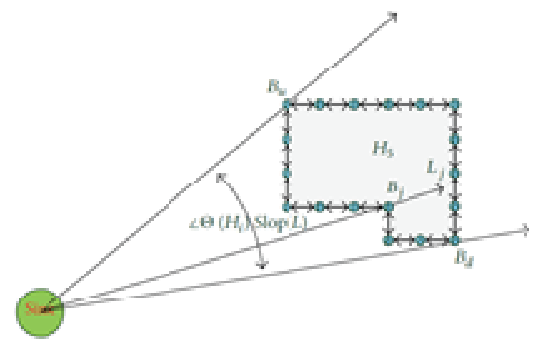

Figure 5. Calculation of Hole Angle.[6]

Now, the metric of hole angle is given by[6]:

$\mathrm{W}\left(\Theta\left(\mathrm{H}_{\mathrm{i}}\right)\right)=\Theta\left(\mathrm{H}_{\mathrm{i}}\right) / 180^{\circ}$;

- Distance Metric: This property is similar to the nearest distance method. As discussed in nearest distance method, nodes closer to the sink have more data to relay. Hence hole closer to the sink will have greater influence on the data transmission than the same hole located far away from the sink. Healing a hole located at a greater distance or located at the edge of the field will only improve coverage and will have very less effect on the connectivity. Therefore, we can say that the influence on the data transmission is inversely proportional to the distance of the hole from the sink. The distance of hole from sink is calculated as[6]:

$d_{i}=\sqrt{\left(H_{i} \cdot x-S_{0} \cdot x\right)^{2}+\left(H_{i} \cdot y-S_{0} \cdot y\right)^{2}}$.

Where $d_{i}$ is the distance of the hole from the sink, $H_{i} \cdot x$ and $H_{i} \cdot y$ are the $x$ and $y$ coordinates of the center of the hole, $S_{0} . x$ and $S_{0} . y$ are the $x$ and y coordinates of the sink. Fig. 6 shows different holes with their distance from the sink. 


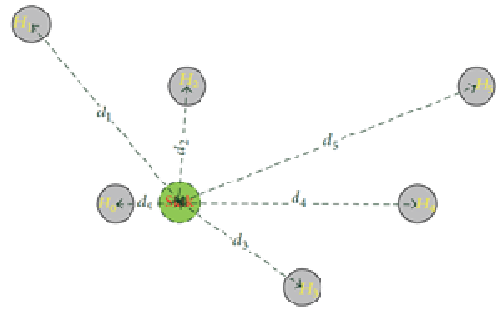

Figure 6. Distance between hole and the sink.[6]

Now the distance metric is given by[6]:

$\mathrm{W}\left(\operatorname{len}\left(\mathrm{H}_{\mathrm{i}}\right)\right)=1 / \mathrm{d}_{\mathrm{i}}$;

- Depth Metric: If a hole area is very large, it will greatly reduce the coverage ratio and might result in network topology disconnection. To avoid this, the depth metric is considered. It is defined as the distance between nearest hole boundary node $B_{\text {near }}$ and farthest hole boundary node $\mathrm{B}_{\mathrm{far}}$, as shown in fig. 7 .

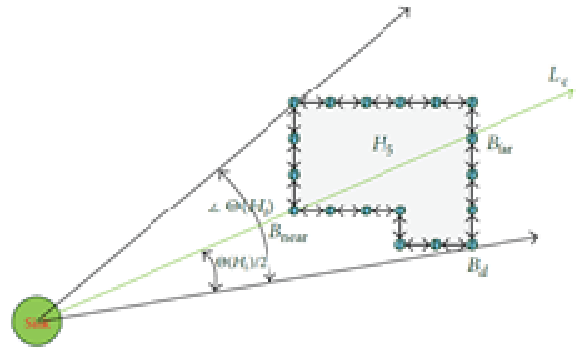

Figure 7. Calculation of Depth.[6]

To calculate $B_{\text {near }}$ and $B_{\text {far }}$ an angle bisector $L_{c}$ of the hole angle $\Theta\left(H_{i}\right)$ is obtained. This angle bisector $\mathrm{L}_{\mathrm{c}}$ makes contact with the hole at two hole boundary nodes i.e. it passes through the transmission range of these two nodes. Out of these two nodes, the node near to the sink is called $\mathrm{B}_{\text {near }}$ and the node far from the sink is called $\mathrm{B}_{\text {far }}$. The calculation for $\mathrm{B}_{\text {near }}, \mathrm{B}_{\text {far }}$ and the depth metric $\mathrm{W}\left(\operatorname{deep}\left(\mathrm{H}_{\mathrm{i}}\right)\right)$ is given as $[6]$ :

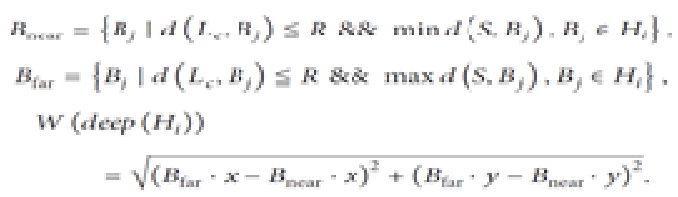

Here, $B_{j}$ represents set of hole boundary nodes, $H_{i}$ represents the hole, $R$ is the transmission radius of sensor nodes, $d(a, b)$ gives the distance between $a$ and $b$.

- These three properties are combined to assign weight to every hole as[6]:

$\mathrm{W}\left(\mathrm{H}_{\mathrm{i}}\right)=\alpha \mathrm{W}\left(\Theta\left(\mathrm{H}_{\mathrm{i}}\right)\right) * \beta \mathrm{W}\left(\operatorname{len}\left(\mathrm{H}_{\mathrm{i}}\right)\right) * \gamma \mathrm{W}\left(\operatorname{deep}\left(\mathrm{H}_{\mathrm{i}}\right)\right)$;

where $\alpha, \beta, \gamma$ are positive harmonic coefficients and their value may vary according to the requirement of the application. These coefficients are defined as: $\alpha+\beta+\gamma=1$; Hole with maximum value for weight i.e. $\mathrm{W}\left(\mathrm{H}_{\mathrm{i}}\right)$ will be selected for healing process.

\subsection{Comparative Study}

The experimental results for Random, Maximum Size, Nearest Distance and Weighted method is given in fig 8, which shows that weighted method performs better than others in terms of average delivery hops. Note that in this graph the results are obtained when weighted method is implemented with equally divided path selection method[6] which will be discussed in section 3 i.e. healing process section. Average Delivery hops is defined as the average number of hops a message has to go through to reach the sink in a wireless sensor network. A decrease in average 
delivery hops means better connectivity, message will take less time to reach sink and it will also reduce energy consumption of the network.

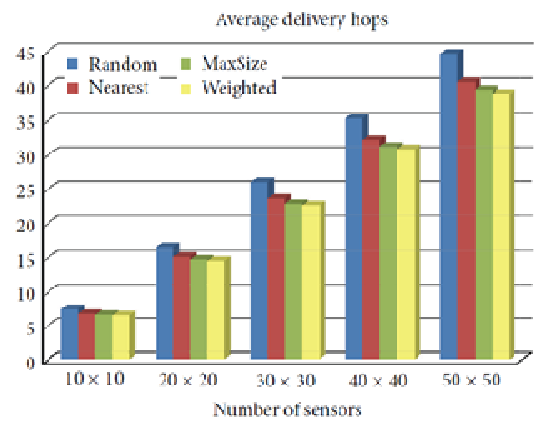

Figure 8. Graph for average delivery hops.[6]

The performance of travelling salesman method is evaluated on the basis of coverage rate[4]. Coverage rate means the percentage of area covered when additional nodes are deployed to cover holes as shown in fig 9. We can see that as we increase the value of additional nodes used for redeployment, the coverage almost reaches $100 \%$. Here[4], Travelling Salesman method is implemented with Delaunay's triangulation method which will be discussed in section 3 i.e. healing process section.

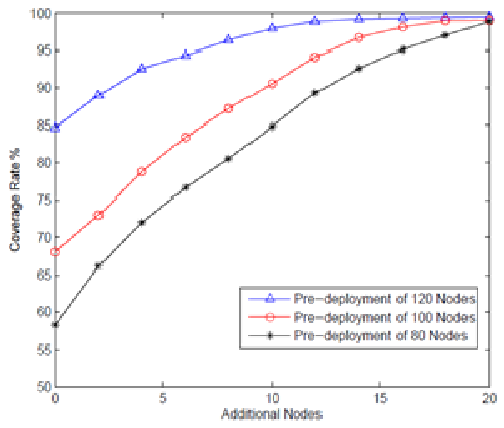

Figure 9. Graph of increase in coverage rate for Travelling Salesman method implemented with Delaunay's triangulation.[4]

The Hole Selection Methods discussed above are compared in table 1 based on their mechanisms and results.

Table 1. Comparative study for hole selection methods.

\begin{tabular}{|l|l|l|}
\hline $\begin{array}{l}\text { Hole Selection } \\
\text { Method }\end{array}$ & Mechanism & Result \\
\hline Random & Hole is selected randomly for healing & Does not optimizes results \\
\hline Maximum Size & Hole with maximum size is selected first & Improves coverage \\
\hline Nearest Distance & Hole closer to the sink is selected first & Improves connectivity \\
\hline $\begin{array}{l}\text { Travelling Salesman } \\
\text { Problem Method }\end{array}$ & $\begin{array}{l}\text { Hole selection is converted into travelling } \\
\text { salesman problem }\end{array}$ & $\begin{array}{l}\text { Covers each and every hole once } \\
\text { within less time. }\end{array}$ \\
\hline Weighted Method & $\begin{array}{l}\text { Hole is selected based on its three } \\
\text { properties i.e. hole angle, distance and } \\
\text { depth. }\end{array}$ & $\begin{array}{l}\text { Improves both connectivity and } \\
\text { coverage. }\end{array}$ \\
\hline
\end{tabular}

\section{Healing Process}

After hole selection, next step is to carry out the healing process. Healing process should aim to maximize the coverage as well as maintain the network topology and connectivity. To heal a 
coverage hole, failed nodes are patched or new nodes are redeployed at the location where coverage is lost. Some healing techniques also aim to maintain the network connectivity by deploying nodes in such a way that the average delivery hops for the messages travelling in the network can be reduced. These healing techniques are discussed as follows:

\subsection{Voronoi Diagram Based Approach}

In wireless sensor networks, voronoi diagram[8][18] is mainly used to detect coverage holes. In this section we will discuss the healing technique using voronoi diagrams for directional sensor networks. A voronoi diagram is constructed by the perpendicular bisectors of the line joining adjacent sensor nodes. These perpendicular bisectors intersect with each other to form a voronoi cell, see fig 10. The voronoi diagram has some properties:

- One voronoi cell will contain exactly one sensor node.

- Any point inside a voronoi cell will be closer to the sensor node of the given voronoi cell than any other sensor node in the network, see fig $10, \mathrm{p}$ is a point inside the voronoi cell of sensor $\mathrm{s} 1$ and the distance between $\mathrm{p}$ and $\mathrm{s} 1$ is shorter than the distance of the point from any other sensor node.

- For a given set of sensor nodes the voronoi diagram generated will be unique[20].

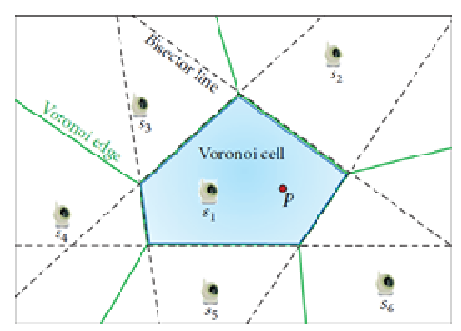

Figure 10. Voronoi Diagram.[2]

Based on these properties, we can say that, for homogeneous sensor nodes with equal sensing range, if a point inside a voronoi cell is not covered by the sensor node of the cell than this point cannot be covered by any other sensor node in the network. Hence, voronoi diagram is also used to detect coverage holes. In [1][2], the redeployment strategy for homogeneous directional sensor nodes using voronoi diagram, to improve the coverage ratio is proposed. The sensing model of directional sensors is shown in fig 11 .

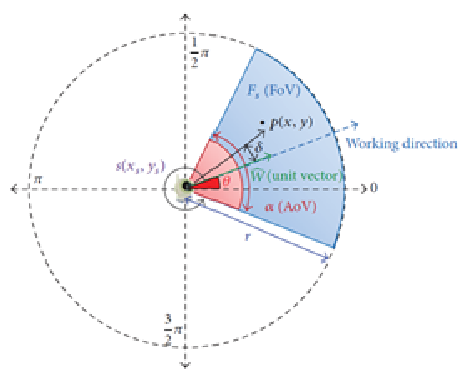

Figure 11. Sensing model of directional sensor.[2]

Here, $\mathrm{s}\left(\mathrm{x}_{\mathrm{s}}, \mathrm{y}_{\mathrm{s}}\right)$ is the sensor, $\mathrm{F}_{\mathrm{s}}($ Field of View, FoV) is the area covered by the sensor, $\alpha$ is called angle of view of the sensor, $\theta$ is the working direction of the sensor and it is measured relative to positive $\mathrm{x}$-axis, $\mathrm{r}$ is the sensing radius, ${ }^{\mathrm{w}} \mathrm{w}$ is the unit vector for working direction of the sensor, $\mathrm{p}(\mathrm{x}, \mathrm{y})$ is a point covered by the sensor ' $\mathrm{s}$ '. $\delta$ is the angle between the vector $\mathrm{sp}^{->}$and unit vector w. 
Based on this sensing model, to cover the point $\mathrm{p}(\mathrm{x}, \mathrm{y})$, the sensor $\mathrm{s}\left(\mathrm{x}_{\mathrm{s}}, \mathrm{y}_{\mathrm{s}}\right)$ has to satisfy the following conditions:

- Distance between ' $p$ ' and 's' should be less than or equals to ' $r$ '.

i.e. $\sqrt{\left(\left(x-x_{\mathrm{s}}\right)^{2}+\left(y-y_{\mathrm{s}}\right)^{2}\right)} \leq \mathrm{r}$;

- The angle ' $\delta$ ' should be less than or equals to half of ' $\alpha$ '.

i.e. $\delta \leq \alpha / 2$;

Considering: $s \vec{p} \cdot \hat{} w=\|s p \vec{w}\| .\|\hat{w}\| \cos \delta$;

$\Rightarrow\left(x-x_{s}\right) \cos \theta+\left(y-y_{s}\right) \sin \theta \geq \sqrt{\left(x-x_{s}\right)^{2}+\left(y-y_{s}\right)^{2}} \cos (\alpha / 2)$;

So far we have discussed the voronoi diagram and the sensing model for directional sensors. Due to the limited sensing range of the directional sensors and random deployment, the target sensing field is not fully covered and it leaves coverage holes in the field as shown in fig 12 . Now we will discuss the hole healing techniques to improve the coverage ratio for directional sensors using voronoi diagram.

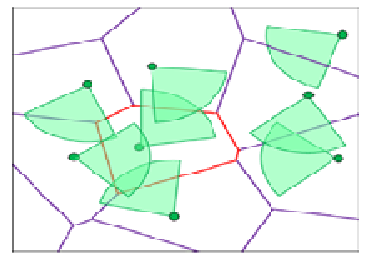

Figure 12. Random deployment of sensor nodes with their voronoi cells.[1]

\subsubsection{Direction Adjustment}

This technique is used for direction rotatable sensors. This means the directional sensors can rotate $360^{\circ}$ and their working direction $\theta$ can be changed. In [1], three algorithms are proposed to adjust the working direction of the sensors such that the overall coverage ratio of the network is improved. Initially the sensor nodes are deployed randomly as shown in fig 12, then each node starts constructing its voronoi cell with the information of its neighbor nodes. After the construction of voronoi cell the three step algorithm works as follows:

- The sensor nodes calculate the area covered by them within their voronoi cell, when they are allowed to face each vertex of the voronoi cell as shown in fig 13. The vertex with maximum coverage area is selected and the working direction of the sensor is rotated towards that vertex.

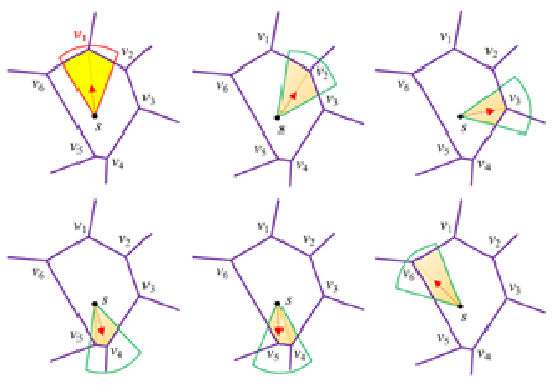

Figure 13. Result of different vertex selection.[1]

During the calculation of the covered area, cases shown in fig 14 can arise. In fig 14a the covered area is equal to the field of view of the sensor and can be calculated as:

$\mathrm{A}_{\mathrm{s}}=(\alpha / 2) \mathrm{r}^{2}$; where $\mathrm{A}_{\mathrm{s}}$ is called Intra-Cell Coverage Area. 
Now the intra-cell coverage area for other cases where field of view of the sensor extends through the cell edges of the voronoi cell as shown in fig $14 \mathrm{~b}, 14 \mathrm{c}, 14 \mathrm{~d}$, is calculated by dividing this area into several triangles. The area of these triangles is summed up to get the final result.

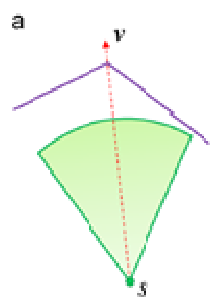
d
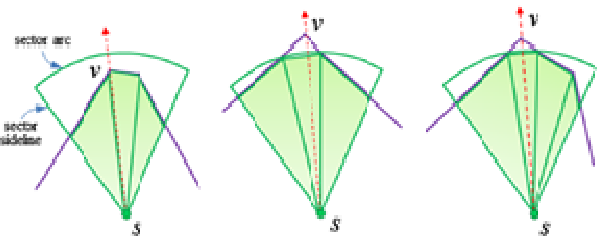

Figure 14. Different cases of Intra-Cell Coverage Area.[1]

To calculate the area of different triangles, first the vertices of triangles are determined. The vertices of triangles include those vertices of voronoi cell which comes inside the range or field of view of the sensor node i.e. those voronoi cell vertices which satisfies eq1 and eq2. Also it includes those points where voronoi cell edges intersect with the sector sideline or sector arc of the field of view of sensor. The equation for the 2 sector sidelines is given as[1]:

$\left(\mathrm{x}-\mathrm{x}_{\mathrm{s}}\right) \cos \theta+\left(\mathrm{y}-\mathrm{y}_{\mathrm{s}}\right) \sin \theta=\sqrt{\left(\mathrm{x}-\mathrm{x}_{\mathrm{s}}\right)^{2}+\left(\mathrm{y}-\mathrm{y}_{\mathrm{s}}\right)^{2}} \cos (\alpha / 2) ;$

where $\sqrt{\left(x-x_{s}\right)^{2}+\left(y-y_{s}\right)^{2} \leq r}$;

The equation for the sector arc is given as[1]:

$\sqrt{\left(\mathrm{x}-\mathrm{x}_{\mathrm{s}}\right)^{2}+\left(\mathrm{y}-\mathrm{y}_{\mathrm{s}}\right)^{2}}=\mathrm{r}$;

where $x \in\left[r \cos (\theta+\alpha / 2)+x_{s}, r \cos (\theta-\alpha / 2)+x_{s}\right]$ and $y \in\left[r \cos (\theta-\alpha / 2)+y_{s}, r \cos (\theta+\alpha / 2)+\right.$ $\mathrm{y}_{\mathrm{s}}$; ;

The equation for the voronoi cell edge is given as[1]:

$\mathrm{x}\left(\mathrm{y}_{\mathrm{a}}-\mathrm{y}_{\mathrm{b}}\right)=\mathrm{y}\left(\mathrm{x}_{\mathrm{a}}-\mathrm{x}_{\mathrm{b}}\right)$;

$\sqrt{\left(x-x_{a}\right)^{2}+\left(y-y_{a}\right)^{2}}+\sqrt{\left(x-x_{b}\right)^{2}+\left(y-y_{b}\right)^{2}}=\sqrt{\left(x_{a}-x_{b}\right)^{2}+\left(y_{a}-y_{b}\right)^{2}}$

where $\left(\mathrm{x}_{\mathrm{a}}, \mathrm{y}_{\mathrm{a}}\right)$ and $\left(\mathrm{x}_{\mathrm{b}}, \mathrm{y}_{\mathrm{b}}\right)$ are two vertices of the voronoi cell edge.

From these equations the intersecting points are obtained. After getting the vertices for triangles, length of the three sides of each triangle is obtained and area of each triangle is calculated as[1]:

$\mathrm{d}=\left(\mathrm{e}_{1}+\mathrm{e}_{2}+\mathrm{e}_{3}\right) / 2$;

Area of triangle $=\sqrt{d\left(d-e_{1}\right)\left(d-e_{2}\right)\left(d-e_{3}\right)}$; where $e_{1}, e_{2}, e_{3}$ are the three sides of the triangle. Now this area for each triangle is summed up to get the intra-cell coverage area when a given voronoi cell vertex is selected. The vertex with maximum value of intra-cell coverage area is selected as the working direction of the sensor. This can be written mathematically as[1]:

$$
\begin{aligned}
& d_{i}^{v}=\frac{1}{2} \sum_{j=1}^{3} e_{i j}^{v}, \quad 1 \leq i \leq k_{v} \\
& v_{D}=\underset{v \in v_{s}}{\operatorname{argmax}} \sum_{i=1}^{k_{v}} \sqrt{d_{i}^{v} \prod_{j=1}^{3}\left(d_{i}^{v}-e_{i j}^{v}\right)}
\end{aligned}
$$

Here $\mathrm{e}_{\mathrm{ij}}^{\mathrm{v}}$ represents the $\mathrm{j}^{\text {th }}$ side of the $\mathrm{i}^{\text {th }}$ triangle when vertex $\mathrm{v}$ of the voronoi cell is selected, $\mathrm{k}_{\mathrm{v}}$ is the total number of triangles for the vertex $\mathrm{v}, \mathrm{V}_{\mathrm{s}}$ is the set of vertices of the given voronoi cell and $\mathrm{v}_{\mathrm{D}}$ is the vertex with maximum intra-cell coverage area. The working direction $\theta$ is calculated as[1]: 


$$
O=f\left(x_{V_{L}}, y_{V_{D}}\right)=\left\{\begin{array}{cl}
\tan ^{-1} \frac{\left(y_{v_{D}}-y_{s}\right)}{\left(x_{v_{D}}-x_{S}\right)}, & x_{V_{D}}>x_{S} \\
\tan ^{-1} \frac{\left(y_{v_{D}}-y_{s}\right)}{\left(x_{v_{D}}-x_{S}\right)}+\pi, & x_{V_{D}}<x_{S} \\
\frac{\pi}{2} \operatorname{sgn}\left(y_{v_{D}}-y_{S}\right), & x_{V_{D}}=x_{S}
\end{array}\right.
$$

- Ine next step is to avold coverage overlap, which can happen if multiple neighboring sensor nodes selects the same common vertex as their working direction as shown in fig 15. Here, sensor node with smaller intra cell coverage area can change its working direction. The condition for this is given as[1]:

$$
\exists s_{i} \in N_{s} \text { such that }
$$

$$
v_{D}^{s}=v_{D}^{s_{i}}, R_{s}^{v_{B}} \cap R_{s_{i}}^{v_{B}} \neq \varnothing \quad \text { and } \quad A_{s}^{v_{s}}<A_{s_{i}}^{v_{B}}
$$

Here, $\mathrm{N}_{\mathrm{s}}$ is the set of neighboring nodes who are adjacent to the voronoi cell of node $\mathrm{s}, \mathrm{v}_{\mathrm{D}}^{\mathrm{s}}$ is the vertex selected as working direction for $\mathrm{s}, \mathrm{R}^{\mathrm{vD}}{ }_{\mathrm{s}}$ is the coverage area or field of view of $\mathrm{s}$ when vertex $\mathrm{v}$ is selected and $\mathrm{A}_{\mathrm{vD}}^{\mathrm{v}}$ is the intra-cell coverage area of $\mathrm{s}$ when vertex $\mathrm{v}$ is selected. Now a new set $\mathrm{U}_{\mathrm{s}}$ of vertices is defined as[1]:

$\mathrm{U}_{\mathrm{s}}=\mathrm{V}_{\mathrm{s}}-\left\{\mathrm{v}_{\mathrm{D}}^{\mathrm{s}}\right\}$; and to avoid overlap the new vertex selected as working direction for $\mathrm{s}$ is given as[1]:

$\mathrm{v}_{\mathrm{D}}^{\prime}=\operatorname{argmax}_{\mathrm{veUs}} \mathrm{A}_{\mathrm{s}}^{\mathrm{v}}$

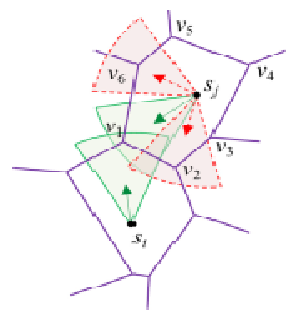

Figure 15. Change in working direction to avoid common vertex and coverage overlap.[1]

In fig 15 , we can see that sensor $\mathrm{s}_{\mathrm{j}}$ changes its working direction from v1 to v2 but still there is some overlap. To avoid this a new set of vertices $U_{s}^{\prime}$ is defined as[1]:

$U_{s}^{\prime}=\left\{v \in U_{s} \| R_{s}^{V} \cap\left(U_{s_{i} \in \hat{S}_{V_{D}}} R_{s_{j}}^{V_{D}}\right)|<| R_{s}^{V_{D}} \cap\left(U_{s_{i}} \in S_{V_{D}} R_{s_{i}}^{V_{D}}\right) \mid\right\}$

This set of vertices includes those vertices which have smaller overlap than $v_{D}$. Here, $S_{v D}$ is the set of those sensor nodes except $s$ whose working direction is $\mathrm{v}_{\mathrm{D}}$ and $\mathrm{U}_{\mathrm{sieSvD}} \mathrm{R}^{\mathrm{vD}}$ si is the union of their coverage area or field of view. Now from set $U_{s}$ overlapped region is subtracted from intracell coverage area of each vertex and the vertex with maximum value is selected for working direction as[1]:

$v_{D}^{\prime}=\operatorname{argmax}_{v \in U_{s}^{r}} A_{s}^{v}-\left|\left(U_{S_{i} \in S_{v_{D}}} R_{S_{i}}^{v_{D}}\right) \cap C_{\xi}^{\mathrm{v}}\right|$

Here, $\mathrm{C}_{\mathrm{s}}^{\mathrm{v}}$ is the intra cell coverage region. As shown in fig 15 working direction is again turned to vertex $v 6$ to avoid the coverage overlap. If every vertex of the voronoi cell is selected by different sensors as working direction, then the vertex with maximum coverage and minimum overlap is selected as[1]:

$v_{D}^{\prime}=\operatorname{argmax}_{v \in V_{s}} A_{s}^{v}-\left|R_{s_{p}}^{v} \cap C_{s}^{v}\right|$

Here $s_{v}$ is the other node which has selected vertex $v$ as working direction.

- The third and last step is to avoid nodes from sensing outside the boundary of the field. Nodes close to the boundary of the field may face towards the boundary which should be avoided. 
If the distance between the boundary of the field and the sensor $\tau$ is less than $\omega r$ where $\omega$ is the coefficient between 0 to 1 then the working direction of the sensor is controlled between the two limits $\theta_{1}$ and $\theta_{2}$ as shown in fig 16 .

i.e. if $\tau<\omega r$; then $0 \leq \theta \leq \theta_{1}$ and $\theta_{2} \leq \theta \leq 2 \pi$;

where $\theta_{1}$ and $\theta_{2}$ is calculated as[1]:

$\theta_{1}=[(\pi-\alpha) / 2]+\sin ^{-1}(\tau / r)$;

$\theta_{2}=[(3 \pi+\alpha) / 2]-\sin ^{-1}(\tau / r)$

Hence in fig 16 the choice for the working direction will be vertex $v_{b}$ and $v_{c}$.

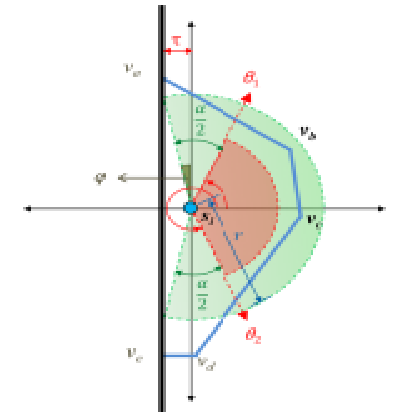

Figure 16. Avoiding coverage outside the boundary.[1]

\subsubsection{Sensor Movement with Direction Adjustment}

As the name suggest this technique is meant for the directional sensor nodes which have been provided mobility. The sensor nodes can change their position and choose an optimal location, from where coverage can be maximized. After getting the right location for movement, the direction of the sensor can be adjusted. Initially sensor nodes are deployed randomly and they start constructing their voronoi cell. Then the sensor node selects one of the voronoi cell vertex as its new location as shown in fig 17.

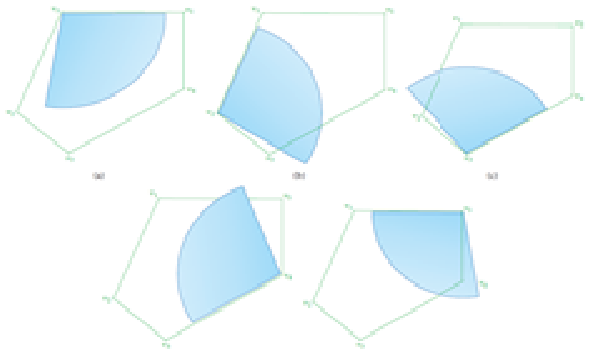

Figure 17. Result of choosing different vertex as location.[2]

The vertex whose angle is greater than angle of view of the sensor and whose edges are greater than sensing radius of the sensor is selected as new location for sensor movement. The angle $\beta$ of the vertex $\mathrm{v}$ is calculated by using the information about its left adjacent vertex $\mathrm{v}_{\mathrm{L}}$ and right adjacent vertex $v_{R}$ as shown in fig 18. The coordinates of these adjacent vertices are $\left(x_{L}, y_{L}\right)$ and $\left(\mathrm{x}_{\mathrm{R}}, \mathrm{x}_{\mathrm{R}}\right)$ respectively and angle $\beta$ is calculated as[2]:

$$
\begin{aligned}
& \beta=\cos ^{-1} \frac{\overrightarrow{w_{i}} \cdot \overrightarrow{w_{R}}}{\left|\overrightarrow{w_{L}}\right|\left|\overrightarrow{v_{R}}\right|} \\
& =\cos ^{-1} \frac{\left(x_{L}-\ddot{x}_{v}\right)\left(x_{R}-x_{v}\right)+\left(y_{L}-y_{v}\right)\left(y_{R}-y_{v}\right)}{\sqrt{\left(x_{L}-x_{v}\right)^{2}+\left(y_{L}-y_{v}\right)} \sqrt{\left(x_{R}-x_{v}\right)^{2}+\left(y_{R}-y_{v}\right)^{2}}}
\end{aligned}
$$




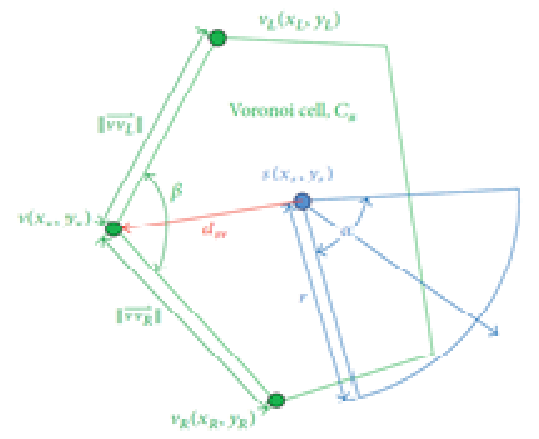

Figure 18. Calculation of the angle $\beta$.[2]

Now a set of vertices $T$, which contains all those vertices of the cell whose angle $\beta$ is greater than or equals to angle of view $\alpha$ and whose edge length is also greater than or equals to sensing radius $r$, is defined as[2]:

$T=\left\{v \in V|\beta \geq \alpha,| \overrightarrow{v v_{L}}\|\geq r,\| \overrightarrow{v v_{R}} \| \geq r\right\}$

From this set $\mathrm{T}$, a target vertex $\mathrm{t}$ is selected with maximum value for $\beta$ as[2]:

$t=\arg \max _{v \in T} \beta$,

If the set $\mathrm{T}$ is empty, then the target vertex is selected among all those vertices having $\beta$ greater than or equals to $\alpha$ and only one edge length greater than or equals to r. If we cannot find any vertex satisfying these conditions then the vertex with largest value for $\beta$ is selected.

Once the sensor is moved to the selected vertex, the working direction $\theta$ of the sensor is adjusted within the limits $\theta_{1}$ and $\theta_{2}$ as shown in fig 19.

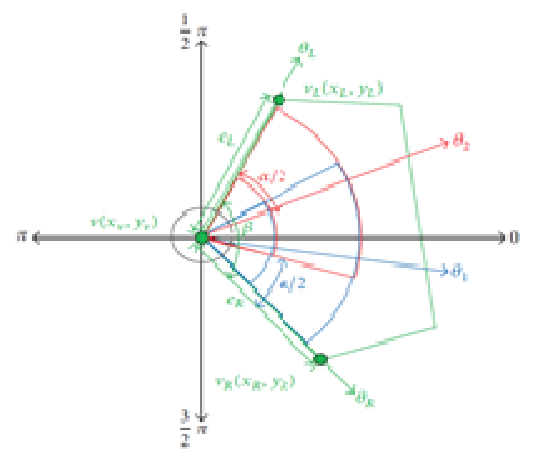

Figure 19. Calculation of the limits $\theta_{1}$ and $\theta_{2}$.[2]

To decide these limits $\theta_{\mathrm{L}}$ and $\theta_{\mathrm{R}}$ [2] are calculated which are defined as the angle of the vector $\mathrm{vv}_{\mathrm{L}}{ }^{->}$and $\mathrm{vv}_{\mathrm{R}}^{->}$with the positive $\mathrm{x}$-axis respectively. $\theta_{1}, \theta_{2}, \theta_{\mathrm{L}}, \theta_{\mathrm{R}}$, [2] all lies between 0 and $+-\pi$.

$$
\theta_{R}=\left\{\begin{array}{c}
\sin ^{-1} \frac{y_{R}-y_{v}}{\sqrt{\left(x_{R}-x_{v}\right)^{2}+\left(y_{R}-y_{v}\right)^{2}}}, \\
\pi-\sin ^{-1} \frac{y_{R}-y_{v}}{\sqrt{\left(x_{R}-x_{v}\right)^{2}+\left(y_{R}-y_{v}\right)^{2}}} \\
x_{R}<x_{v}, y_{R}>y_{v} \\
-\pi-\sin ^{-1} \frac{y_{R}-y_{v}}{\sqrt{\left(x_{R}-x_{v}\right)^{2}+\left(y_{R}-y_{v}\right)^{2}}} \\
x_{R}<x_{v}, y_{R}<y_{v}
\end{array}\right.
$$$$
\theta_{L}=\left\{\begin{array}{c}
\sin ^{-1} \frac{y_{L}-y_{v}}{\sqrt{\left(x_{L}-x_{v}\right)^{2}+\left(y_{L}-y_{v}\right)^{2}}}, \\
\pi-\sin ^{-1} \frac{y_{L}-y_{v}}{\sqrt{\left(x_{L}-x_{v}\right)^{2}+\left(y_{L}-y_{v}\right)^{2}}}, \\
x_{L}<x_{v}, y_{L}>y_{v} \\
-\pi-\sin ^{-1} \frac{y_{L}-y_{v}}{\sqrt{\left(x_{L}-x_{v}\right)^{2}+\left(y_{L}-y_{v}\right)^{2}}}, \\
x_{L}<x_{v}, y_{L}<y_{v} .
\end{array}\right.
$$ 
If the sensor is rotated towards the right side edge $e_{R}$ of the vertex $v$ then $\theta_{1}$ will be the working direction of the sensor and if sensor is rotated towards left side edge $e_{L}$ of the vertex $v$ then $\theta_{2}$ will be the working direction.

$\theta_{1}=\left\{\begin{array}{ll}\theta_{R}+\frac{\alpha}{2}, & \theta_{R}+\frac{\alpha}{2} \leq \pi \\ \theta_{R}+\frac{\alpha}{2}-2 \pi, & \text { otherwise }\end{array} \quad \theta_{2}= \begin{cases}\theta_{L}-\frac{\alpha}{2}, & \theta_{L}-\frac{\alpha}{2} \geq-\pi \\ \theta_{L}-\frac{\alpha}{2}+2 \pi, & \text { otherwise. }\end{cases}\right.$

To improve the coverage ratio, sensor will be rotated towards the longest edge as shown in fig 20 .
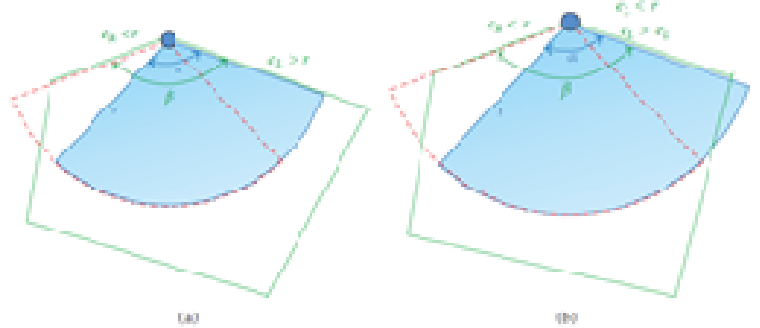

Figure 20. Cases of rotation of sensor.[2]

Let $\theta_{0}$ is the initial working direction of the sensor when it is moved to the vertex $\mathrm{v}$, then the extent or range $\varphi$ to which $\theta_{0}$ should be rotated in either clockwise or anticlockwise direction within the limits $\theta_{1}$ and $\theta_{2}$ is given as[2]:

$\varphi= \begin{cases}\left\{\begin{array}{ll}\left|e_{0}-\theta_{1}\right|, & \left|\theta_{0}-\theta_{1}\right| \leq \pi \\ 2 \pi-\left|\theta_{0}-\theta_{1}\right|, & \left|\theta_{0}-\theta_{1}\right|>\pi\end{array}, \quad e_{R} \geq e_{L}\right. \\ \left\{\begin{array}{ll}\left|e_{0}-\theta_{2}\right|, & \left|\theta_{0}-\theta_{2}\right| \leq \pi \\ 2 \pi-\left|\theta_{0}-\theta_{2}\right|, & \left|\theta_{0}-\theta_{2}\right|>\pi\end{array}, e_{L}>e_{R} .\right.\end{cases}$

\subsection{Triangular Diagram Based Approach}

In this technique every hole is healed by a single sensor node. A new node is deployed in the region where hole is detected. In this process first the hole is detected then area of that hole is calculated and thereafter the healing process starts. To heal a hole the area of the hole is required. Area of the hole is calculated with the help of triangular diagram. This diagram is obtained by joining the center points of every adjacent sensor node which as a result forms a triangle for every three adjacent nodes in the network, see fig 21.

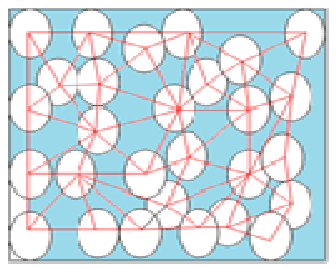

Figure 21. Triangular structure.[3]

Depending on the location of adjacent sensor nodes and distance between them several cases may arise as shown in fig 22. Here, we can see the location of hole and intersection between adjacent sensor nodes. $s_{1}, s_{2}$ and $s_{3}$ is the area of intersection between the sensor's sensing region and the triangle. 
International Journal of Ad hoc, Sensor \& Ubiquitous Computing (IJASUC) Vol.7, No.1, February 2016

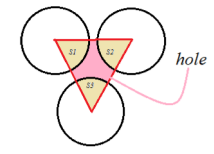

(a)

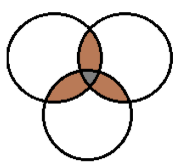

(f)

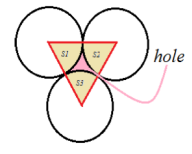

(b)

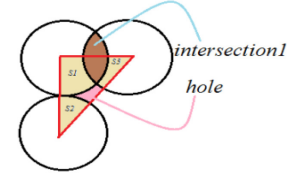

(g)

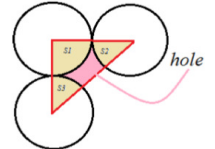

(c)

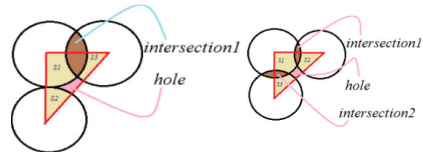

(d)

(e)

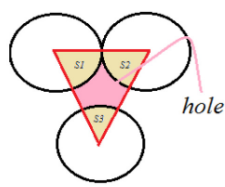

(h)

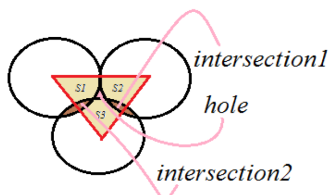

(i)

Figure 22. All cases for three adjacent sensors.[3]

In order to calculate the area of the hole, we have to do some calculations first. Let a,b,c be the three sides of the triangles shown in different cases in fig 22. Then the area of these triangles represented by $\mathrm{s}_{\Delta}$ is calculated as[3]:

$\mathrm{s}_{\Delta}=(1 / 4) \sqrt{(\mathrm{a}+\mathrm{b}+\mathrm{c})(\mathrm{a}-\mathrm{b}+\mathrm{c})(\mathrm{b}-\mathrm{a}+\mathrm{c})(\mathrm{c}-\mathrm{a}+\mathrm{b})} ;$

and the relationship between region $\mathrm{s}_{1}, \mathrm{~s}_{2}$ and $\mathrm{s}_{3}$ is given as[3]:

$s_{1}+s_{2}+s_{3}=(1 / 2) \pi R^{2}$; where $R$ is the sensing radius.

Now the area of the intersection of two circles or nodes represented by $s_{\text {intersect }}$ is calculated as shown in fig 23 . Here, two circles with different radius $\mathrm{R}, \mathrm{r}$ and location $(0,0),(\mathrm{d}, 0)$ respectively are considered.

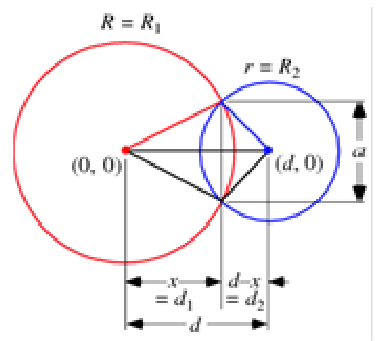

Figure 23. Calculation for the area of intersection of two circles.[3]

From fig 23, area of the intersection represented by $\mathrm{A}$ is given as[3]:

$A=A\left(R, d_{1}\right)+A\left(r, d_{2}\right)$

$=r^{2} \cos ^{-1}\left(\frac{d^{2}+r^{2}-R^{2}}{2 d r}\right)+R^{2} \cos ^{-1}\left(\frac{d^{2}-r^{2}+R^{2}}{2 d R}\right)$

$-\frac{1}{2} \sqrt{(d+r-R)(-d+r+R)(d-r+R)(d+r+R)}$

where $A\left(r, d_{2}\right)$ is the area of left portion of the intersection divided by line segment ' $a$ ' and $A(R$, $\mathrm{d}_{1}$ ) is for the right portion. $\mathrm{d}_{1}$ and $\mathrm{d}_{2}$ is given as[3]:

$d_{1}=x=\frac{d^{2}-r^{2}-R^{2}}{2 d} \quad d_{2}=d-x=\frac{d^{2}+r^{2}-R^{2}}{2 d}$

Now in fig 22 sensor nodes are homogeneous, therefore here $r=R$ and area of hole $s_{h}$ for all these cases is thus calculated as[3]:

- For fig $22 \mathrm{a}, 22 \mathrm{~b}, 22 \mathrm{c}, 22 \mathrm{~h}$ area is given by[3]:

$\mathrm{s}_{\mathrm{h}}=\mathrm{s}_{\Delta}-\left(\mathrm{s}_{1}+\mathrm{s}_{2}+\mathrm{s}_{3}\right)$; 
- For fig 22d,22g area is given by[3]:

$$
\begin{aligned}
& \mathrm{s}_{\mathrm{h}}=\mathrm{s}_{\Delta}-\left(\mathrm{s}_{1}+\mathrm{s}_{2}+\mathrm{s}_{3}\right)+(1 / 2) \mathrm{s}_{\text {intersect }} \text {; and } \\
& S_{\text {int er sec } t}=R^{2} \cos ^{-1}\left(\frac{d^{2}}{2 d R}\right)-R^{2} \cos ^{-1}\left(\frac{d^{2}}{2 d R}\right)-\frac{1}{2} \sqrt{(-d+2 R) d^{2}(d+2 R)}
\end{aligned}
$$

- $\quad$ For fig $22 \mathrm{e}$ area is given by[3]:

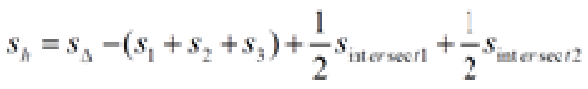

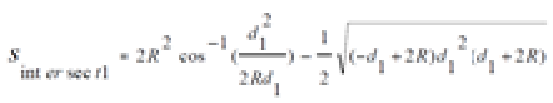

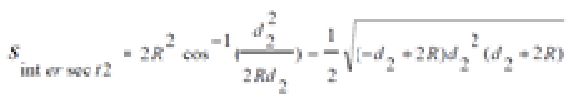

- In fig $22 \mathrm{f}$, hole doesn't exist.

- For fig $22 \mathrm{i}$ area is given by[3]:

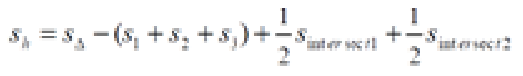

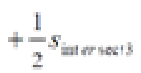

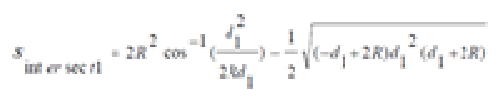

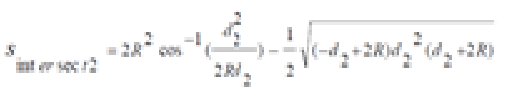

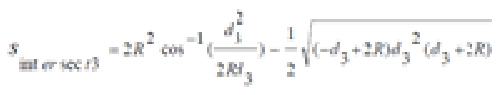

After calculating area of the hole, healing process starts. Each hole is healed by a single sensor node. New sensor nodes are deployed in uncovered regions by following two methods:

- If the sensing area of the sensor is greater than area of the hole than circumcircle methods is used as shown in fig 24 . The circumcircle is obtained by the perpendicular bisectors of the three sides of the triangle. The point where these perpendicular bisectors intersect is called the circumcircle center of the triangle and this is the point where new nodes is deployed.

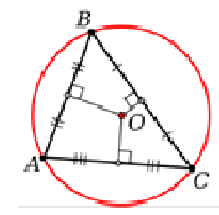

Figure 24. Calculation for circumcircle center of the triangle.[3]

- If the sensing area of the sensor is less than the area of the hole than incircle method is used as shown in fig 25.The incircle is obtained by the angle bisectors of the three angles of triangle. The point where these three angle bisectors intersect is called incircle center of the triangle and this is the point where new node is deployed. The incircle is inscribed inside the triangle and it is tangent to each side of the triangle. 


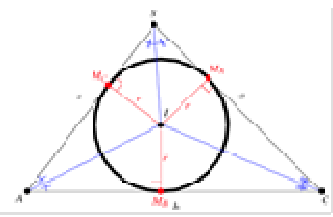

Figure 25. Calculation for incircle center of the triangle.[3]

\subsection{Delaunay's Triangulation}

Delaunay's Triangulation[8][18][19] is a geometrical structure which is formed by a given set of points. It is a triangulation method in which triangles are formed in such a way that circumcircle of each triangle will not contain any other point of the given set. This means the vertices of the triangle will lie on the circumference of the circle and the circle will be empty inside. In fig 26(a), the triangulation formed is Delaunay's triangulation because each circumcicle is empty and 26(b) is not Delaunay's triangulation because each cicumcircle encloses a point inside it. Also the minimum angle of a triangle formed by Delaunay's Triangulation is maximum for other triangulations for the same set of points.
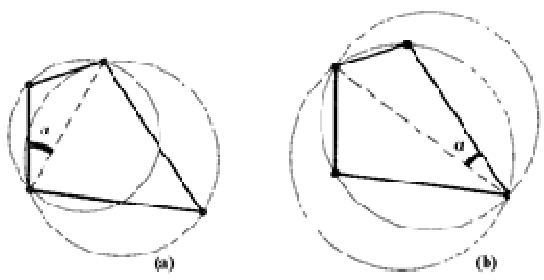

Figure 26. Triangulation for a given set of four points. The minimum angle $\alpha$ shown in (a) is greater than the minimum angle shown in (b).[8]

$\operatorname{In}[4]$, an algorithm is proposed to heal coverage holes using Delaunay's triangulation as shown in fig 27. Holes with irregular shapes and boundaries are analyzed and the point where the irregular curve turns is considered as a vertex. All these vertices together form a closed polygon and then the Delaunay's triangulation is performed over the polygon vertices. The radius for the circumcircle of the triangles formed is calculated and new node is deployed at the center of the circumcircle whose radius is maximum.

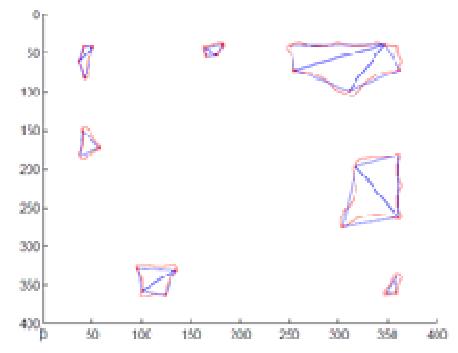

Figure 27. Delaunay Triangulation of holes.[4]

\subsection{Equally Divided Path Selection Method}

This method says that new nodes should be patched along the angle bisector of the hole angle discussed in section 2.5 for healing the hole. This path is the shortest path for data to travel to the sink. To prove this, a hole $\mathrm{H}_{\mathrm{i}}$ is considered inside a parallelogram TUVW as shown in fig 28 . A and $\mathrm{B}$ are two nodes equidistant and symmetrical from the sink. Let $\mathrm{q}$ is a point on $\mathrm{AB}$ and $\mathrm{p}$ is a point on $\mathrm{TU}$ such that $\mathrm{Aq}=\mathrm{x}, \mathrm{Bq}=\mathrm{y}, \mathrm{Ap}=\mathrm{a}, \mathrm{Bp}=\mathrm{b}$ and the distance between line $\mathrm{AB}$ and $\mathrm{p}$ i.e. $\mathrm{qp}=\mathrm{h}$. Now using the property of a right angled triangle[6]:

$\mathrm{a}^{2}=\mathrm{h}^{2}+\mathrm{x}^{2}$;

$\mathrm{b}^{2}=\mathrm{h}^{2}+\mathrm{y}^{2}$; 
Let a function $\mathrm{f}(\mathrm{x})=\mathrm{a}+\mathrm{b}$; and $\mathrm{AB}=\mathrm{x}+\mathrm{y}=\mathrm{L}$;

$=>\mathrm{f}(\mathrm{x})=\sqrt{\mathrm{h}^{2}+\mathrm{x}^{2}}+\sqrt{\mathrm{h}^{2}+(\mathrm{L}-\mathrm{x})^{2}}$;

First and Second order derivative of $\mathrm{f}(\mathrm{x})$ is given as[6]:

$$
\begin{aligned}
f^{\prime}(x) & =\frac{x}{\left(x^{2}+h^{2}\right)^{1 / 2}}-\frac{L-x}{\left[(L-x)^{2}+h^{2}\right]^{1 / 2}}, \\
f^{\prime \prime}(x)= & \frac{\left(x^{2}+h^{2}\right)^{1 / 2}+x \times(1 / 2)\left(x^{2}+h^{2}\right)^{-1 / 2}}{\left(x^{2}+h^{2}\right)} \\
& -\left(\left[(L-x)^{2}+h^{2}\right]^{1 / 2} \times(-1)+(L-x)\right. \\
& \left.\times \frac{1}{2}\left(x^{2}+h^{2}\right)^{-1 / 2} \times(-2)(L-x)\right) \\
& \times\left[(L-x)^{2}+h^{2}\right]^{-1} .
\end{aligned}
$$

Now by using maximum value theorem, the minimum value of $x$ for the function $f(x)$ comes out to be $\mathrm{L} / 2$. This means minimum value for $a+b$ will be obtained when $x$ and $y$ are equal i.e. $q$ lies in the center and passes through the angle bisector. Hence, the shortest path to reach sink through the hole will be along the angle bisector. New nodes will be patched along this path.

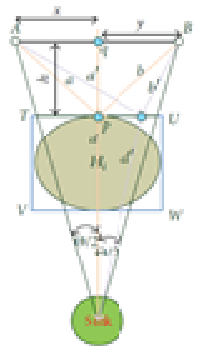

Figure 28. Calculation for shortest path along the angle bisector.[6]

\subsection{Comparative Study}

The experimental results for direction adjustment and sensor movement with direction adjustment is given in fig 29 and fig 30. The results shows that out of these two voronoi diagram based healing techniques, sensor movement with direction adjustment is better. The results are obtained for sensor nodes with angle of view $\alpha=120^{\circ}$ and sensing radius $=50$ metres and field size $=500 \mathrm{~m} * 500 \mathrm{~m}$. This shows that providing sensor nodes with moving capabilities helps in improving overall coverage of the network.

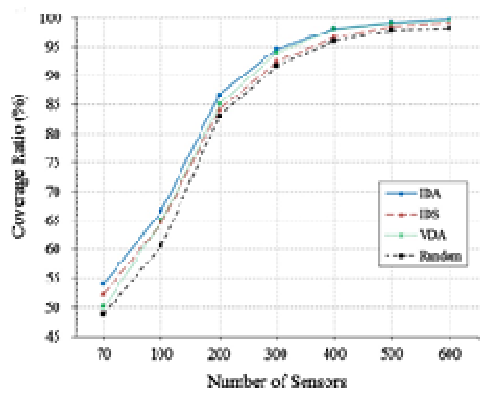

Figure 29. IDA represents Direction Adjustment.[1] 


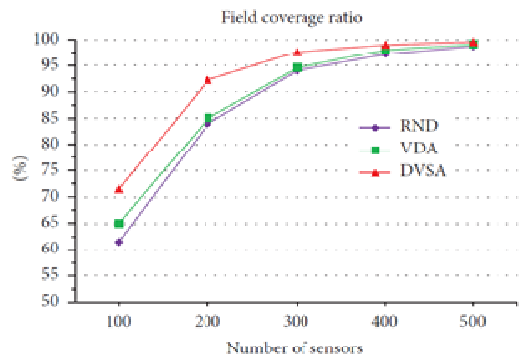

Figure 30. DVSA represents Sensor Movement with Direction Adjustment.[2]

The graph for Delaunay's triangulation and equally divided path selection method is discussed earlier in section 2.6. with fig 9 and fig 8, where they are implemented along with travelling salesman method and weighted method. The equally divided path selection method is evaluated in terms of average delivery hops whereas other methods are evaluated based on coverage ratio. This is because equally divided path selection method focus on improving connectivity by providing shorter routes to the active nodes in the network. A comparative study of these methods is given in table 2 .

Table 2. Comparative study for healing process methods.

\begin{tabular}{|c|c|c|c|c|}
\hline \multicolumn{2}{|c|}{ Healing Process Method } & Mechanism & Utility & Type of Sensor \\
\hline \multirow{2}{*}{$\begin{array}{l}\text { Voronoi } \\
\text { Diagram } \\
\text { Based } \\
\text { Approach }\end{array}$} & $\begin{array}{l}\text { Direction } \\
\text { Adjustment }\end{array}$ & $\begin{array}{l}\text { Voronoi cell vertex is selected } \\
\text { as working direction of the } \\
\text { sensor. }\end{array}$ & \multirow{2}{*}{$\begin{array}{l}\text { Can be used } \\
\text { for both hole } \\
\text { detection and } \\
\text { Healing }\end{array}$} & \multirow[t]{2}{*}{$\begin{array}{l}\text { Directional } \\
\text { Sensor Network }\end{array}$} \\
\hline & $\begin{array}{l}\text { Sensor } \\
\text { Movement with } \\
\text { Direction } \\
\text { Adjustment } \\
\end{array}$ & $\begin{array}{l}\text { Voronoi cell vertex is selected } \\
\text { as the new location for the } \\
\text { sensor. }\end{array}$ & & \\
\hline \multicolumn{2}{|c|}{$\begin{array}{l}\text { Triangular Diagram Based } \\
\text { Approach }\end{array}$} & $\begin{array}{l}\text { New node is deployed either on } \\
\text { circumcircle center or incircle } \\
\text { center of the triangle, based on } \\
\text { the size of the hole. }\end{array}$ & $\begin{array}{l}\text { Hole } \\
\text { Detection and } \\
\text { Healing }\end{array}$ & $\begin{array}{l}\text { Omni- } \\
\text { Directional }\end{array}$ \\
\hline \multicolumn{2}{|c|}{$\begin{array}{l}\text { Delaunay's Triangulation } \\
\text { Method }\end{array}$} & $\begin{array}{l}\text { Hole is divided into triangles } \\
\text { and new node is deployed on } \\
\text { circumcircle center of the } \\
\text { largest triangle. }\end{array}$ & Hole Healing & $\begin{array}{l}\text { Omni- } \\
\text { Directional }\end{array}$ \\
\hline \multicolumn{2}{|c|}{$\begin{array}{l}\text { Equally divided path selection } \\
\text { Method }\end{array}$} & $\begin{array}{l}\text { New nodes are deployed along } \\
\text { the angle bisector of the hole } \\
\text { angle. }\end{array}$ & Hole Healing & $\begin{array}{l}\text { Omni- } \\
\text { Directional }\end{array}$ \\
\hline
\end{tabular}

\section{CONCLUSION}

In this study, we discussed about various coverage problems and hole healing strategies in WSN. It can be noticed that with the heterogeneity in sensor nodes we get different type of coverage problems and hence will need different strategies to overcome these problems e.g. in mobile sensor networks, coverage is improved by moving the sensors to appropriate locations, in directional sensor networks, coverage is improved by adjusting the sensing directions of these sensors, and in static sensor networks, we can patch failed nodes or can use redeployment methods. The coverage holes can be healed in a random order or a proper sequence can be followed. This sequence can be obtained by converting the problem into travelling salesman problem, covering each hole once and returning back to the initial hole[4]. Priority healing is another option where holes can be assigned a priority value based on some properties of the hole[6]. All these techniques have their own advantages and disadvantages. Some algorithms are centralized while others are distributed. The centralized algorithms results in optimal routes while poses communication overhead. The distributed algorithms may not result in optimal routes but 
International Journal of Ad hoc, Sensor \& Ubiquitous Computing (IJASUC) Vol.7, No.1, February 2016

they are energy efficient and can be used for scalable networks. Random healing result in increasing the coverage ratio only while priority healing not only increases coverage but it can also reduce energy consumption of the network during the healing process. Keeping in mind all these factors we can choose an appropriate healing method suitable for our application.

\section{REFERENCES}

[1] T.-W. Sung and C.-S. Yang, (2013) "Voronoi-based coverage improvement approach for wireless directional sensor networks", Journal of Network and Computer Applications.

[2] T.-W. Sung and C.-S. Yang, (2013) "Distributed voronoi-based self-redeployment for coverage enhancement in a mobile directional sensor network," International Journal of Distributed Sensor Networks, Article ID 165498, 15 pages.

[3] S. Babaie and S. S. Pirahesh, (2012) "Hole detection for increasing coverage in wireless sensor network using triangular structure,” IJCSI International Journal of Computer Science Issues, vol. 9, no. 1 , article 2 .

[4] P. Si, C. Wu, Y. Zhang, Z. Xia, and L. Cheng, (2011) "A hole detection and redeployment strategy in wireless sensor network," Journal of Information and Computational Science, vol. 8, no. 13, pp.25772585 .

[5] C. Zhang, Y. Zhang, and Y. Fang, (2010) "A coverage inference protocol for wireless sensor networks," IEEE Transactions on Mobile Computing, vol. 9, no. 6, pp. 850-864.

[6] Fu-Tian Lin, Tien-Wen Sung, and Chu-Sing Yang, (2013) "A Coverage Hole Healing Strategy with Awareness of Data Delivery Time in Wireless Sensor Networks," International Journal of Distributed Sensor Networks, Volume 2013, Article ID 790794, 11 pages.

[7] Waltenegus Dargie and Christian Poellabauer (2010) Fundamentals of Wireless Sensor Networks, Theory and Practice; A John Wiley and Sons, Ltd., Publication.

[8] F. Aurenhammer, (1991) "Voronoi diagrams-a survey of a fundamental geometric data structure," ACM Computing Surveys, vol. 23, no. 3, pp. 345-405.

[9] Junbin LIANG, Ming LIU and Xiaoyan KUI, (2014) "A Survey of Coverage Problems in Wireless Sensor Networks,” Sensors \& Transducers, Vol. 163 , Issue 1, pp. 240-246.

[10] J. Yick, B. Mukherjee and D.Ghosal, (2008) "Wireless sensor network survey," Computer Networks, vol. 52, no. 12, pp. 2292-2330.

[11] F. Ye, G. Zhong, S. Lu and L. Zhang, (2002) "PEAS, a robust energy conserving protocol for long lived sensor networks," in Proceedings of 10th IEEE International Conference on Network Protocols (ICNP'02), Paris, France, pp. 200-201.

[12] D. Tian and N. D. Georganas, (2003) "A node scheduling scheme for energy conservation in large wireless sensor networks," Wireless Communications and Mobile Computing, Vol. 3, Issue 2, pp. 271-290.

[13] J. Wu and S. Yang, (2004) "Coverage issue in sensor networks with adjustable ranges," in Proceedings of International Workshop on Mobile and Wireless Networking (MWN'04) in conjunction with International Conference on Parallel Processing Workshops (ICPPW'04), Montreal, Quebec, Canada, pp. 61-68.

[14] M. Cardei, M. Thai, Y. Li, and W. Wu, (2005) "Energy efficient target coverage in wireless sensor networks," in Proceedings of the 24th Conference of the IEEE Communications Society (INFOCOM'05), pp. 1976-1984. 
International Journal of Ad hoc, Sensor \& Ubiquitous Computing (IJASUC) Vol.7, No.1, February 2016

[15] Hang Ding Xing, Zhang Bo Yun and Tang Wen Shen, (2009) "Distributed connected algorithm for wireless sensor networks," Computer Engineering and Application, Vol. 45, Issue 7, pp. 17-19.

[16] I. Vasilescu, K. Kotay, D. Rus, M. Dunbabin and P. Corke, (2005) "Data collection, storage, retrieval with an underwater sensor network," in: Proceedings of the Third International Conference on Embedded Networked Sensor Systems (Sensys), SanDiego, CA.

[17] Smita Karmakar and Alak Roy, (2014) "Holes Detection in Wireless Sensor Networks: A Survey," I.J. Modern Education and Computer Science, 4, 24-30

[18] D. Du, F. Hwang, S. Fortune, (1992) "Voronoi diagrams and Delaunay triangulations," Euclidean Geometry and Computers.

[19] ChunHsien Wu, KuoChuan Lee, YehChing Chung, (2006) “A Delaunay Triangulation Based Method for Wireless Sensor Network Depoyment," In Proceedings of the 12th International Conference on Parallel and Distributed Systems, pp. 1521-1530

[20] A. Okabe, B. Boots, and K. Sugihara, (2000) Spatial Tessellations, Concepts and Applications of Voronoi Diagrams, John Wiley \& Sons, 2nd edition.

\section{AUTHORS}

Latesh Mehta is pursuing his Master degree in Computer Science Engineering from Chitkara University, Himachal Pradesh, India. He is enrolled in Integrated Bachelor + Master Degree course in Computer Science Engineering from Chitkara University, Himachal Pradesh, India. His main research area includes, Wireless Sensor Network.

Manik Gupta is Assistant Professor in the Department of Computer Science at Chitkara University, Himachal Pradesh, India. He has a good number of publications in the field of wireless sensor networks in various international conferences and journals of good repute. He had been awarded for Best Research Paper, in December, 2010. He also worked as Software Developer for one year after completing his Bachelors in Engineering and as Consultant in LSI, Research and Development. He also remained a regular columnist of

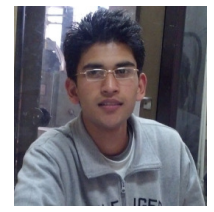
column "Manik's Tech Tonics" in the newspaper- "Student Age". His research area is Security, Energy Efficiency, Fault Tolerance, Fault Revoking, Coverage, Connectivity and Mobility in Wireless Sensor Networks and Body Area Sensor Networks. 